HISPANIA. Revista Española de Historia, 2009, vol. LXIX, núm. 231, enero-abril, págs. 209-234, ISSN: 0018-2141

\title{
LA EVOLUCIÓN DE LA ESTATURA EN UNA REGIÓN ATRASADA DE LA ESPAÑA INTERIOR: CASTILLA Y LEÓN, 1830-1960*
}

\author{
JAVIER MORENO LÁZARO \\ Universidad de Valladolid \\ José M. MARTíneZ CARRIÓN
}

Universidad de Murcia

RESUMEN: Este artículo desea contribuir al desarrollo de la bistoria antropométrica que ba suscitado el interés reciente de los historiadores sociales y económicos. A partir del análisis de la estatura masculina en la España interior, aporta evidencias del estado nutricional y el bienestar biológico en la era de la industrialización española. Con datos de los reemplazos militares se construyen series de una muestra de las poblaciones urbanas y rurales de Castilla-León, compuesta por casi 29000 registros de las cohortes de 1830-1940, se analizan el «trend» secular, los ciclos y las desigualdades territoriales, ambientales y sociales. Los resultados revelan que la evolución de las estaturas estuvo sometida a ciclos condicionados por factores ambientales y económicos, que la talla disminuyó a mediados del siglo XIX ante situaciones de malnutrición y no se recuperó hasta las generaciones nacidas a finales del siglo $X I X$. El deterioro de la talla fue más ostensible en los ambientes urbanos y entre los jornaleros. La mejora del estado nutricional fue significativa en la primera mitad del siglo XX, y aunque recibió el impacto de la Guerra Civil y la posguerra, éste no fue tan acusado como en otras regiones de España.

Javier Moreno Lázaro es profesor en la Universidad de Valladolid. Dirección para correspondencia: Departamento de Fundamentos del Análisis Económico e Historia e Instituciones Económicas, Facultad de Económicas y Empresariales, Avda. Valle de Esgueva, 47011,Valladolid.E-mail: jmoreno@eco.uva.es

José M. Martínez Carrión es catedrático en la Universidad de Murcia. Dirección para correspondencia: Departamento de Economía Aplicada, Facultad de Economía y Empresa, Campus de Espinardo, Universidad de Murcia,30100,Murcia.E-mail: jcarrion@um.es

* Este trabajo ha sido realizado en el marco de los Proyectos BEC2002-03927 y SEJ200767613/ECON, financiados por el MEC y el Ministerio de Ciencia e Innovación (Gobierno de España) respectivamente. Versiones preliminares de este texto han sido presentadas al XIV Congreso Internacional de Antropología Biológica de la AEAB (Murcia, 19-22 de octubre de 2005) y al III Congreso Internacional de Historia Económica de la AMHE, celebrado en Cuernacava-Morelos (México), 29-31 de octubre de 2007. Los autores agradecen los comentarios de los participantes en dichas conferencias a las primeras versiones y, asimismo, de los evaluadores de la revista. 
Palabras Clave: Historia antropométrica. Bienestar biológico. Estatura. Nivel de vida. Castilla y León. Siglos XIX y XX.

\section{EvOlution OF THE STATURE IN A BACKWARD REGION OF SPAIN: CASTILE AND LEON, $1830-1960$}

ABSTRACT: This article contributes to the field of anthropometric history, recently of great interest to social and economic historians. An analysis of the height of adult males in Spain's interior provides a reflection of the nutritional state and biological wellbeing of the society of that region during Spain's industrialization. Some 29,000 registers relating to military drafts between 1830 and 1940 provide a series of samples of the rural and urban populations of Castilla-León which allow for an analysis of territorial, environmental, and social inequalities, as well as cyclical changes. This analysis reveals that height in this region developed according to a cyclical model determined by environmental and economic factors, diminishing during the second balf of the nineteenth century, when malnutrition and under-nutrition were more prevalent in Castilla-León than in other Spanish regions, and not picking up again before the early twentieth century. This reduction in height was more evident in urban environments and among day-labourers. The nutritional status of this region's population improved significantly during the first half of the twentieth century. Although it was affected during the Civil War and post-war period, this decline was not as great as that experienced in other regions of Spain.

KEY WORDS: Anthropometric history. Biological well-being. Height. Standard of living. Secular trend. Castile and Leon. $19^{\text {th }}$ and $20^{\text {th }}$ centuries.

\section{INTRODUCCIÓN}

En los últimos años ha aumentado el interés de los historiadores económicos y sociales por el explorar la evolución de los niveles de vida y el bienestar de las poblaciones españolas ${ }^{1}$. La cuestión ha tenido un amplio eco por parte de la denominada 'historia antropométrica': un campo de estudio que explora el nivel de vida a partir de indicadores antropométricos, como la estatura y el peso a determinadas edades y en diferentes contextos socioeconómicos y ambientales $^{2}$. De acuerdo con las investigaciones biomédicas, la estatura media

1 Bolos, Jordi, JARne, A. y ViCEDo, EnRIC (eds.), Condiciones de vida al món rural. V Congrés sobre sistemas agraris, organització social i poder local, Lleida, Institut d'Estudis Ilerdencs \& Diputació de Lleida, 2006; EsCUDERO, Antonio y SimÓN, Hipólito, «El bienestar en España: una perspectiva de largo plazo, 1850-1991», Revista de Historia Económica, XXI/3 (2003), págs. 593-621; MARTínEZ CARrión, José Miguel (ed.), El nivel de vida en la España rural, siglos XVIII-XX, Publicaciones de la Universidad de Alicante, 2002.

2 Los primeros estudios de antropometría histórica se deben a la escuela francesa de Emmanuel Le Roy Ladurie, siendo pionero el trabajo de ArOn, Jean-Paul, DumONT, Paul y Le RoY LADURIÈ, Emmanuel, Anthropologie du conscrit français 1819-1826, París, Mouton, 1972. Sobre la evolución de la historia antropométrica y el papel de la estatura como indicador del bienestar y el nivel de vida, véanse KOMLOS, John, «¿Qué es la historia antropométrica?», Revista de Historia Económica, XII/3 (1994), págs. 781-786; KomLOS, John (ed.), Stature, living standard, and economic 
que una población determinada alcanza en un momento histórico concreto estaría determinada por la experiencia nutritiva de los individuos que componen esa población, siendo decisivas las condiciones de la infancia y de la adolescencia. El contexto epidemiológico y ambiental desempeñaría, asimismo, un papel determinante en la evolución de la ingesta nutritiva que influye finalmente en el proceso de crecimiento de la altura física y del bienestar humano. Además del consumo de alimentos, principalmente de nutrientes y energía, el impacto de las enfermedades y las condiciones de vida y trabajo tendrían una influencia apreciable en la configuración de la salud física y del estado nutricional y, por tanto, en la estatura media final de las poblaciones, que como se sabe, se alcanza en torno a los 18-20 años.

El avance de los estudios de historia antropométrica ha sido significativo en España, al menos en la última década. Aunque los primeros trabajos se dieron a conocer a mediados de la década de $1980^{3}$, en la última década se han dado a conocer resultados sólidos que han aportado evidencias interesantes para el debate sobre los niveles de vida en España. Hoy son ya varias decenas de publicaciones las que se han centrado sobre la evolución de la talla de los españoles y que abordan los cambios seculares desde mediados del siglo XIX hasta la actualidad. Aunque la mayoría han tenido como principal objetivo evaluar el impacto del desarrollo económico en el largo plazo sobre el bienestar y los niveles de vida biológicos de la población española ${ }^{4}$, tampoco han faltado estudios antropométricos que han planteado la cuestión en los momentos finales del Antiguo Régimen ${ }^{5}$. Con miles de datos de estaturas masculinas, principal-

development. Essays in anthropometric history, Chicago, The University of Chicago Press, 1994; KomLos, John (ed.), The biological standard of living on three continents. Further explorations in anthropometric history, Boulder, Westview Press, 1995; Komlos, John y BaTeN, Jörg (eds.), The Biological Standard of Living in Comparative Perspective, Stuttgart, Franz Steiner Verlag, 1998; Komlos, John y BATEN, Joerg (eds.), Special Issue: Recent research in Anthropometric History, Social Science History, 28/2 (2004), págs. 191-350.

3 Gómez Mendoza, Antonio y Pérez Moreda, Vicente, «Estatura y nivel de vida en la España del primer tercio del siglo XX», Moneda y Crédito, 174 (1985), págs. 29-64; MARTíneZ CARRIÓN, José M., «Estatura, nutrición y nivel de vida en Murcia, 1860-1930», Revista de Historia Económica, IV/1, (1986), págs. 67-99.

4 Martínez Carrión, José Miguel, «El nivel de vida en la España rural, siglos XVIII-XX. Nuevos enfoques, nuevos resultados», en: MARTínez CARrión, José Miguel (ed.), El nivel de vida en la España rura..., págs. 16-72; MARTínez CARrión, José Miguel, «Biología, historia y medio ambiente. La estatura como espejo del nivel de vida de la sociedad española», Ayer. Revista de Historia Contemporánea, 46 (2002), págs. 93-122; MARTÍnEZ CARRIÓN, José Miguel, «El nivel de vida y el bienestar en la España contemporánea. Nuevas aportaciones», en: BOLOS, Jordi, JARNE, A. y VICEDO, ENRIC (eds.), Condiciones de vida al món rural. $V$ Congrés sobre sistemas agraris, organització social i poder local, Lleida, Institut d'Estudis Ilerdencs \& Diputació de Lleida, 2006, págs. 255-285; ReBATO, Ester, «The studies on secular trend in Spain: A review», en: BoDZsÁr, B.E, y SusanNe, C. (eds.), Secular growth changes in Europe, Budapest, Eötvös University Press, 1998, págs. 297-317.

5 CÁmara Hueso, Antonio David, «Fuentes documentales antropométricas para la España rural, 1770-1912», Historia Agraria, 38 (2006), págs. 103-118. 
mente de mozos llamados a filas, en periodo de reclutamiento y servicio militar, la reciente historiografía española ha documentado momentos de deterioro del bienestar humano a partir de la caída de la talla media de los mozos llamados a filas (quintos) y de los reclutas en los comienzos de la industrialización o, si se prefiere, en las primeras fases del crecimiento económico moderno que los especialistas han versado para las décadas centrales del siglo XIX; también se ha proporcionado evidencia que pone de manifiesto la existencia de ciclos en la tendencia secular (secular trend) de la talla media durante el siglo XX —en particular, se advierte un deterioro del estado nutricional entre los mozos y reclutas que vivieron la Guerra Civil y la larga posguerra-; y, por último, se han observado notorias diferencias urbano-rurales, junto a desigualdades sociales, económicas, profesionales y ambientales.

Los resultados antropométricos evidencian que en el curso del siglo XX hubo importantes mejoras del bienestar humano y del nivel de vida biológico. Los estudios han mostrado que las mejoras de la renta, el consumo alimenticio y la salud han posibilitado, junto a los progresos del nivel de vida material en general, un aumento significativo de la talla de los españoles. Esta incrementó un promedio de aproximadamente 12 centímetros durante el curso del siglo XX, al pasar de $163 \mathrm{~cm}$, altura media estimada hacia 1900 entre los reclutas de 20 años de edad, a $175 \mathrm{~cm}$ al final de la década de $1990^{6}$. Probablemente el incremento más significativo alcanzado en la talla de los españoles en el curso de su larga trayectoria biológica.

Los progresos del bienestar humano han sido desiguales en el espacio y el tiempo. No todas las regiones han presentado la misma tasa de crecimiento en las estaturas medias. Así, la desigualdad económica interregional, tan acusada en España antes de la integración del país en la Unión Europea tuvo su réplica en las estaturas. Los datos sugieren fuertes contrastes regionales que merecen el interés de los historiadores para las etapas anteriores a la modernización definitiva de la economía y sociedad españolas. Un ejemplo: para la talla de las generaciones que habían nacido en los años previos a la Guerra Civil de 1936-39 las diferencias entre las estaturas medias regionales fueron de hasta casi 5 centímetros, estableciéndose en torno a $164 \mathrm{~cm}$ en las regiones agrarias y más atrasa-

6 Martínez Carrión, José Miguel, «Niveles de vida y desarrollo económico en la España contemporánea. Una visión antropométrica», Revista de Historia Económica, 3 (1994), págs. 685-716; MARTíNEZ CARrión, José Miguel, «Estaturas, desigualdad regional y desarrollo económico en Italia y España durante el siglo XX», Mediterráneo Económico, Monográfico: Mediterráneo e Historia Económica, 7 (2005), págs. 206-228; MARTínez CARrión, José Miguel, «Calidad de vida y bienestar biológico en el largo plazo. El caso de España», en: DOBADO, Rafael, GómEZ Galvarriato, Aurora y MÁrquez, Graciela (eds.), México y España. ¿Historias económicas paralelas?, México D.F., Fondo de Cultura de México, 2007, págs. 673-706; QuiroGA, Gloria, «Estatura, diferencias regionales y sociales y niveles de vida en España (1893-1954)", Revista de Historia Económica, XIX/1 (2001), págs. 175-200. 
das y en 168-169 cm en las regiones más industrializadas y avanzadas en su desarrollo económico.

El objetivo del presente trabajo es evaluar el bienestar humano y el nivel de vida biológico, de acuerdo con la expresión acuñada por Komlos (1993), lo cual se explora a partir de la talla de los quintos en la Comunidad de Castilla y León, región que en la España contemporánea ha formado parte de las áreas económicamente menos afortunadas. Identificada con los territorios de la Cuenca del Duero, Castilla y León fue el corazón político del imperio y el motor del crecimiento demográfico y económico de la Corona de Castilla y de los reinos peninsulares en los siglos XV y XVI. Con el siglo XVII comienza una pérdida paulatina de su poderío económico, pese a que presenció un tímido progreso a fines del siglo XVIII y desarrolló, tras la Revolución Liberal, un modelo de crecimiento económico de «capitalismo agrario», basado en la agricultura extensiva de secano y en la explotación de sus productos, pero incapaz de garantizar el progreso económico en el devenir del siglo $\mathrm{XX}^{7}$. El planteamiento del ejercicio antropométrico realizado es preguntarse qué impacto tuvo el precitado modelo de crecimiento económico en el crecimiento físico y, asimismo, mostrar la importancia de la estatura de las poblaciones como indicador del nivel de vida biológico y el bienestar humano ${ }^{8}$. En este sentido, el artículo analiza la evolución de la estatura media desde los primeros reemplazos universales, implantados desde 1858, y explora aspectos sociales y ambientales que dicho modelo de crecimiento ejerció sobre el bienestar biológico de las poblaciones castellano y leonesas.

7 García SANZ, Ángel, «Indagaciones sobre las causas históricas de la actual situación de atraso relativo de la economía de Castilla y León», Papeles de Economía Española, 20 (1984), págs. 333-349; MORENO LÁZARO, Javier, «La precaria industrialización de Castilla y León», en: GERMÁN, Luis, Llopis, Enrique, MALUQuer De Motes, Jordi y ZaPATA, Santiago (eds.), Historia Económica Regional de España, Siglos XIX y XX, Barcelona, Crítica, 2001, págs. 182-208.

8 De extraordinaria utilidad son los trabajos de ENGERMAN, Stanley L., "The standard of living debate in international perspective: measures and indicators», en: STECKEL, R.H. y FLOUD, R.C. (eds.), Health and welfare during industrialization, Chicago, The University of Chicago Press, 1997, págs. 17-45; SvedBerg, Peter, Poverty and undernutrition. Theory, measurement, and policy. With a foreword by Amartya Sen, Oxford, Oxford University Press, 2000; FlOUD, R.C., «The heights of Europeans since 1750: a new source for European economic history», en: KomLOS, John (eds.), Stature, living standards, and economic development, Chicago, University of Chicago Press, 1994, págs. 9-24; Floud, R.C., WATCHeR, K.W. y GReGORY, A.S., Height, health and history: Nutritional status in Britain, 1750-1980, Cambridge, Cambridge University Press, 1990; FoGEL, R.W., «Nutrition and the decline in mortality since 1700: some preliminary findings», en: ENGERMAN, S.L. y Gallman R.E. (eds.), Long-term factors in American economic growth, The University of Chicago Press, Chicago, 1986, págs. 439-555; FOGEL, R.W. «El crecimiento económico, la teoría de la población y la fisiología: la influencia de los procesos a largo plazo en la elaboración de la política económica», Revista de Historia Económica, XII/3 (1994), págs. 719- 762. 


\section{MATERIAL Y MÉTODO. LA COMPOSICIÓN DE LA MUESTRA}

Los datos de estatura de la muestra provienen de las Actas de Reclutamiento y Reemplazo que se encuentran en los Archivos Municipales de los ayuntamientos, en las correspondientes secciones de Quintas. El primer reemplazo universal, que registra al conjunto de los mozos nacidos en un determinado municipio, independientemente de su condición social y económica, data de 1858. El estudio se prolonga hasta el reemplazo de 1969, año en que cambió el registro de estaturas, pasando de milímetros a centímetros.

La muestra está compuesta por 28945 mozos que fueron tallados en 14 municipios (Tabla 1), pertenecientes a cuatro provincias del norte de la Comunidad de Castilla y León (Burgos, Palencia, Valladolid y Zamora). La elección de las localidades estudiadas responde a características afines al tipo de hábitat, la especialización productiva y las singularidades orográficas de la región. Incluye tres capitales de provincia (Burgos, Palencia y Zamora), dos localidades ubicadas en la Montaña Palentina (Alar del Rey y Aguilar de Campoo), otras tantas situadas en Aliste (San Vitero y Ferreruela de Tábara), una comarca zamorana limítrofe con Portugal extremadamente atrasada, Fuentesaúco, villa también en Zamora, y seis municipios de la Tierra de Campos.

La Tabla 2 muestra las principales características socio-profesionales de los mozos estudiados, en su mayor parte urbanos. No hubo cambios significativos en los niveles de actividad a lo largo del período analizado. Las principales actividades manufactureras a mediados del XIX y comienzos del XX fueron la industria textil lanera en Palencia y Astudillo, la harinera y galletera en Aguilar y Alar del Rey, la papelera y curtidora en Burgos; y la alcoholera y chocolatera en Zamora. En las localidades escogidas se dan prácticamente todas las variantes de producción agraria conocida en la región, determinadas por la orografía, los recursos hídricos y la disponibilidad de medios de transporte: la agricultura de subsistencia en la Montaña Palentina y Aliste, la horticultura muy mercantilizada en Fuentesaúco y la agricultura extensiva de secano en la Tierra de Campos.

Además del fuerte componente urbano que tiene la muestra, el mayor inconveniente se advierte, como ha reiterado la literatura especializada, en los cambios producidos en la edad de reclutamiento: 1858 (20 años), 1885 (19 años), 1901 (20 años) y 1907 (21 años). Sin embargo, las Actas de Reclutamiento y Reemplazo permiten medir la distorsión que introducen los cambios normativos en los cálculos, en tanto que los mozos momentáneamente excluidos eran tallados durante tres años consecutivos después de la primera medición hasta (en su caso) ser exonerados de participar en los sorteos o ingresar directamente en filas. Los resultados se muestran en la Tabla 3, que presenta el crecimiento anual medio de unos 1200 mozos palentinos y zamoranos. Las estimaciones sobrestiman el incremento anual de la altura de los jóvenes, ya que la mayor parte de quienes se sometían a nuevas mediciones en alistamientos posteriores al suyo eran cortos de talla, por debajo de $156 \mathrm{~cm}$, mucho más propensos a crecer. 
MAPA 1. DISTRIBUCIÓN GEOGRÁFICA DE LAS LOCALIDADES QUE COMPONEN LA MUESTRA

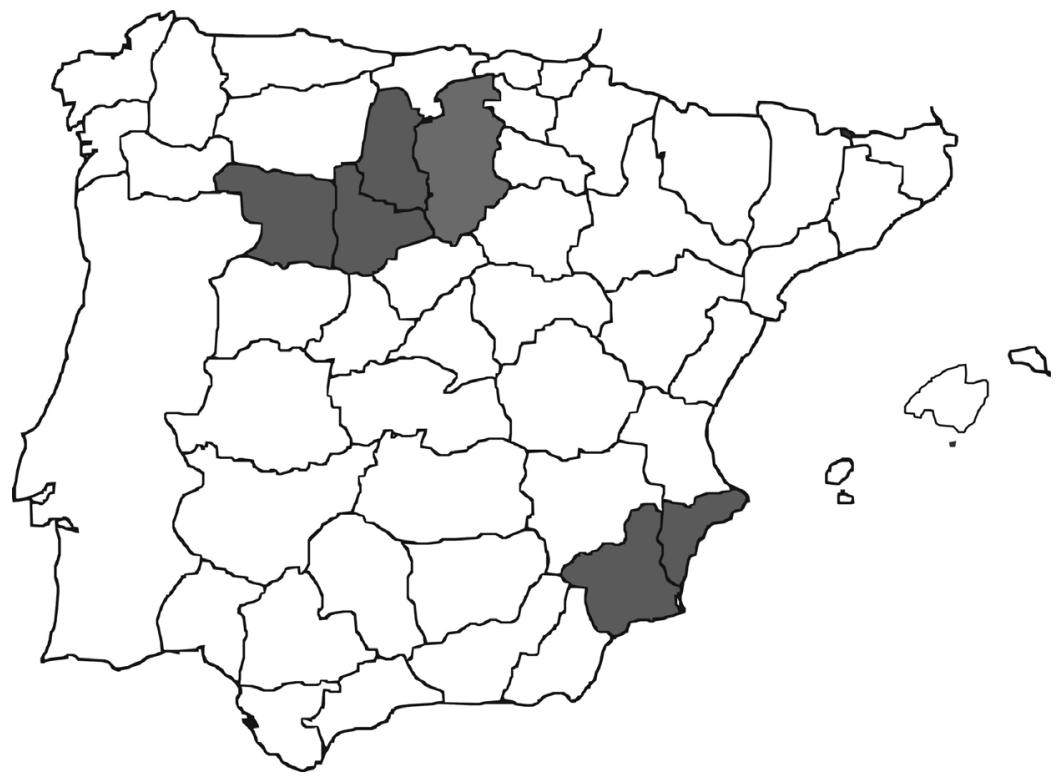

TABLA 1. LOCALIDADES Y OBSERVACIONES QUE COMPONEN LA MUESTRA

\begin{tabular}{|l|c|c|c|c|}
\hline Localidad & Provincia & Período & Mozos llamados & Mozos medidos \\
\hline Aguilar de Campoo & Palencia & $1858-1957$ & 891 & 753 \\
\hline Alar del Rey & Palencia & $1862-1969$ & 810 & 669 \\
\hline Astudillo & Palencia & $1859-1969$ & 2.809 & 2.740 \\
\hline Burgos & Burgos & $1859-1871$ & 2.028 & 1.604 \\
\hline Carrión de los Condes & Palencia & $1960-1969$ & 169 & 142 \\
\hline Ferreruela de Tabarra & Zamora & $1929-1935$ & 89 & 70 \\
\hline Fuentesaúco & Zamora & $1862-1925$ & 1.367 & 1.581 \\
\hline Palacios del Alcor & Palencia & $1901-1939$ & 75 & 60 \\
\hline Palencia & Palencia & $1859-1948$ & 10.649 & 9.075 \\
\hline Medina de Rioseco & Valladolid & $1859-1949$ & 3.996 & 3.199 \\
\hline San Vitero & Zamora & $1921-1939$ & 203 & 128 \\
\hline Villada & Palencia & $1859-1889$ & 490 & 483 \\
\hline Villafácila & Zamora & $1881-1924$ & 349 & 320 \\
\hline Zamora & Zamora & $1859-1948$ & 10.041 & 8.121 \\
\hline TOTAL & & & 33.966 & 28.945 \\
\hline
\end{tabular}

Fuente: Actas de Reclutamiento 
TABLA 2. CARACTERÍSTICAS DE LA MUESTRA

\begin{tabular}{|c|c|c|c|}
\hline & $1858-1899$ & $1901-1936$ & 1937-1969 \\
\hline Mozos llamados & 14.090 & 16.289 & 3.587 \\
\hline Localidades & 9 & 12 & 8 \\
\hline Provincias & 4 & 3 & 3 \\
\hline Mozos urbanos (\%) & 73,4 & 77,8 & 43,9 \\
\hline Mozos rurales $(\%)$ & 26,5 & 22,1 & 56,0 \\
\hline Mozos expósitos ${ }^{(a)}$ & 16,1 & 5,7 & n.d. \\
\hline Mozos activos $(\%)$ & $89,2^{(\mathrm{b})}$ & 89,3 & 89,7 \\
\hline Mozos ocupados en el sector agrario (\%) & $36,6^{(\mathrm{b})}$ & 29,21 & 56,1 \\
\hline
\end{tabular}

(a): Datos correspondientes a la ciudad de Zamora

(b): Dato correspondiente al período 1878-1899

Fuente: Actas de Reclutamiento

TABLA 3. INCREMENTO DE LA TALLA OBSERVADA EN LOS JÓVENES DE LA CIUDAD DE PALENCIA, AL PASAR DE LOS 19 A LOS 23 AÑOS, REEMPLAZOS DE 1858-1936 (EN MILÍMETROS Y PORCENTAJE SOBRE LA TALLA MEDIA)

\begin{tabular}{|c|c|c|c|c|c|c|}
\hline \multirow{2}{*}{ EDAD } & \multicolumn{2}{|c|}{$1858-1885$} & \multicolumn{2}{c|}{$1885-1899$} & \multicolumn{2}{c|}{$1900-1936$} \\
\cline { 2 - 7 } & \multicolumn{2}{|c|}{ Incremento } & \multicolumn{2}{c|}{ Incremento } & \multicolumn{2}{c|}{ Incremento } \\
\cline { 2 - 7 } & Absoluto & Relativo & Absoluto & Relativo & Absoluto & Relativo \\
\hline 19 a 21 & n.d. & n.d. & n.d. & n.d. & 4,2 & 2,6 \\
\hline 19 a 20 & 6,4 & 4,0 & 8,9 & 5,5 & n.d. & n.d. \\
\hline 20 a 21 & 5 & 3,1 & 7,1 & 4,4 & 5,7 & 3,5 \\
\hline 21 a 22 & n.d. & n.d. & n.d. & n.d & 5 & 3,1 \\
\hline 20 a 22 & n.d. & n.d. & n.d. & n.d & 6,1 & 3,7 \\
\hline 20 a 23 & n.d. & n.d. & n.d. & n.d & 2,1 & 1,3 \\
\hline
\end{tabular}

Fuente: Expedientes de revisión de talla.

Con tales características, el artículo analiza la tendencia secular de la talla que, luego, es comparada con la serie del sureste de España, muestra las diferencias urbano-rurales, según residencia de los mozos y analiza, también, las diferencias sociales, a tenor de las profesiones. Con ello, se explora la desigualdad social y la evolución del nivel de vida biológico. Finalmente, se señalan algunas conclusiones que son relevantes para las diferentes etapas del bienestar humano a lo largo del periodo analizado de 110 años, que abarca las generaciones nacidas de 1838 a 1948 . 


\section{LA EVOLUCIÓN DE LA ESTATURA O DEL NIVEL DE VIDA BIOLÓGICO EN CASTILlA Y LEÓN. RESUlTADOS Y DISCUSIÓN}

La Figura 1 y la Tabla 4 presentan los resultados de los promedios quinquenales de la talla de los mozos castellanos y leoneses en el periodo considerado, por años de nacimiento y años de reclutamiento a edades fijadas en los llamamientos. La Figura 2 muestra la evolución de la talla media estandarizada a la edad de 21 años y subsana, por tanto, los sesgos que comportan los cambios en la edad de reclutamiento haciendo uso de las estimaciones incluidas en el Cuadro 3. Los datos evidencian, de una parte, una tendencia secular de crecimiento del bienestar humano en el largo plazo y, de otra, la existencia de ciclos que alteran el ritmo de crecimiento de dicha tendencia, registrándose su mayor deterioro a mediados del siglo XIX.

Figura 1. TALla MEDIA QUINQUENAL DE LA MUESTRA POR COHORTES DE NACIMIENTO SEGÚN LA EDAD DE RECLUTAMIENTO, 1839-1948

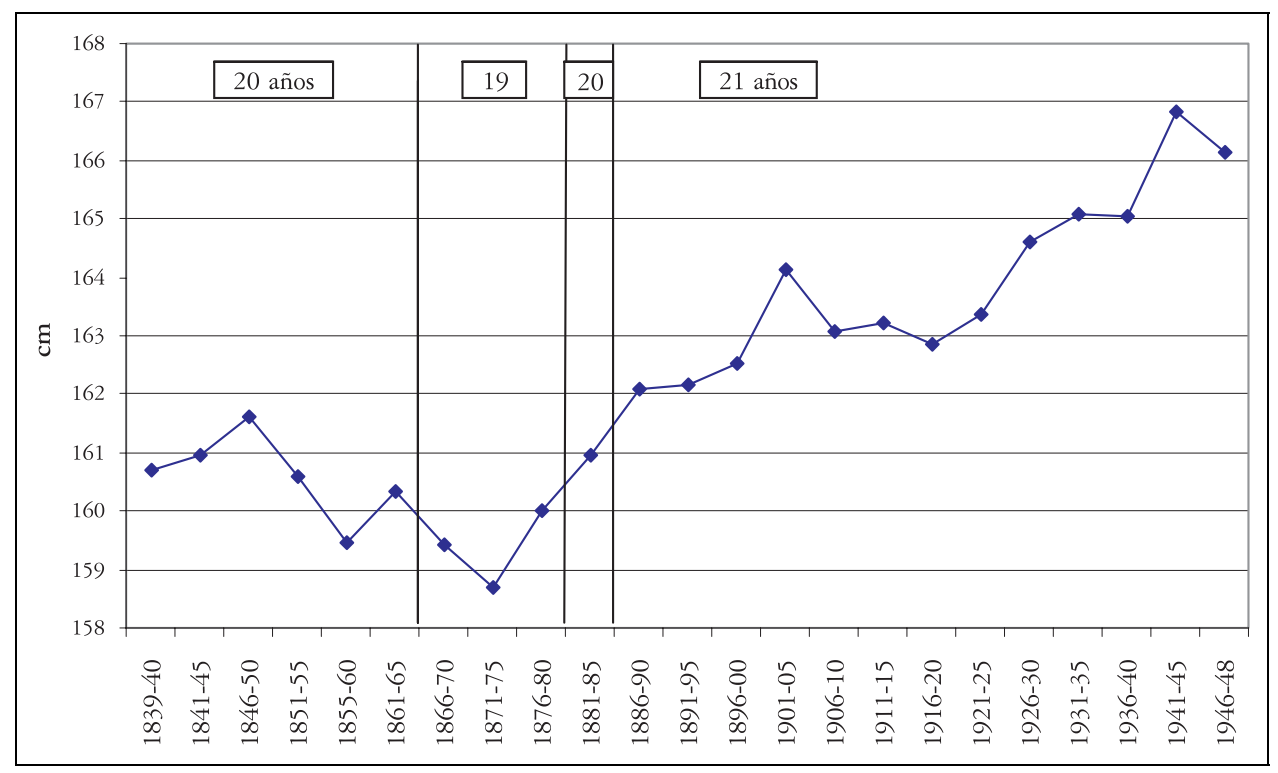

En el largo plazo, la talla media de los mozos castellanos y leoneses creció casi $5 \mathrm{~cm}$, si tomamos como referencia los promedios de las cohortes de 183840 y 1946-48, con estimaciones de estaturas medias de $161,2 \mathrm{~cm}$ y $166 \mathrm{~cm}$ respectivamente. El crecimiento fue notorio si se toma como referencia un periodo más corto, el de las cohortes de 1855-60, que bajó la talla por ser años críticos, y el 1941-45; entre ambos la estatura media creció casi 7 cm (Figura 
2). Pero, como se ha dicho, este crecimiento estuvo jalonado por épocas de retroceso y avances. Veamos a continuación su evolución por etapas.

TABla 4. ESTATURA MEDiA DE LOS MOZOS CASTELLANOS Y LEONESES, POR QUINQUENIOS, ENTRE LOS REEMPLAZOS DE 1858 A 1969 (COHORTES NACIDAS ENTRE 1838 Y 1848)

\begin{tabular}{|c|c|c|c|c|c|c|}
\hline $\begin{array}{c}\text { Período } \\
\text { Reemplazo }\end{array}$ & $\begin{array}{c}\text { Año de } \\
\text { nacimiento }\end{array}$ & $\begin{array}{c}\text { Mozos } \\
\text { llamados }\end{array}$ & $\begin{array}{c}\text { Mozos } \\
\text { tallados }\end{array}$ & Cortos & $\begin{array}{c}\text { Cortos } \\
\% \text { Total }\end{array}$ & Talla media \\
\hline $1858-60$ & $1838-40$ & 742 & 632 & 108 & 17,1 & 160,71 \\
\hline $1861-65$ & $1841-45$ & 2.171 & 1.872 & 309 & 16,5 & 160,94 \\
\hline $1866-70$ & $1846-50$ & 2.132 & 1.825 & 237 & 13,0 & 161,60 \\
\hline $1871-75$ & $1851-55$ & 1.292 & 1.050 & 196 & 18,7 & 160,61 \\
\hline $1875-80$ & $1856-60$ & 1.787 & 1.501 & 356 & 23,7 & 159,45 \\
\hline $1881-85$ & $1861-65$ & 1.770 & 1.472 & 288 & 19,6 & 160,35 \\
\hline $1885-89$ & $1866-70$ & 1.695 & 1.555 & 381 & 24,5 & 159,43 \\
\hline $1890-94$ & $1871-75$ & 1.872 & 1.687 & 461 & 27,3 & 158,71 \\
\hline $1895-99$ & $1876-80$ & 2.086 & 1.882 & 439 & 23,3 & 160,02 \\
\hline $1901-05$ & $1881-85$ & 1.841 & 1.541 & 287 & 18,6 & 160,97 \\
\hline $1907-11$ & $1886-90$ & 2.101 & 1.810 & 239 & 13,2 & 162,09 \\
\hline $1912-16$ & $1891-95$ & 2.063 & 1.612 & 232 & 14,4 & 162,16 \\
\hline $1917-21$ & $1896-00$ & 2.012 & 1.526 & 183 & 12,0 & 162,53 \\
\hline $1922-26$ & $1901-05$ & 2.274 & 1.765 & 194 & 11,0 & 164,13 \\
\hline $1927-31$ & $1906-10$ & 2.313 & 1.871 & 194 & 10,4 & 163,06 \\
\hline $1932-36$ & $1911-15$ & 2.637 & 2.134 & 192 & 9,0 & 163,21 \\
\hline $1937-41$ & $1916-20$ & 894 & 481 & 47 & 9,8 & 162,84 \\
\hline $1942-46$ & $1921-25$ & 1.185 & 966 & 56 & 5,8 & 163,38 \\
\hline $1947-51$ & $1926-30$ & 808 & 642 & 38 & 5,9 & 164,60 \\
\hline $1952-56$ & $1931-35$ & 171 & 153 & 2 & 1,3 & 165,08 \\
\hline $1957-61$ & $1936-40$ & 196 & 182 & 7 & 3,8 & 165,04 \\
\hline $1962-66$ & $1941-45$ & 265 & 219 & 5 & 2,3 & 166,84 \\
\hline $1967-69$ & $1946-48$ & 155 & 131 & 9 & 6,9 & 166,00 \\
\hline
\end{tabular}

Fuente: elaboración propia a partir de las Actas de Reclutamiento de los municipios correspondientes: Aguilar de Campoo, Alar del Rey, Astudillo, Carrión de los Condes, Palacios del Alcor, Palencia y Villada, (de la provincia de Palencia); Burgos (Burgos); Ferreruela de Tabarra, Fuentesaúco, San Vitro, Villafácila y Zamora (Zamora) y Medina del Rioseco (Valladolid). 
FIGURA 2. TALLA MEDIA QUINQUENAL POR COHORTES DE NACIMIENTO ESTANDARIZADA A LA EDAD DE 21 AÑOS, 1839-194

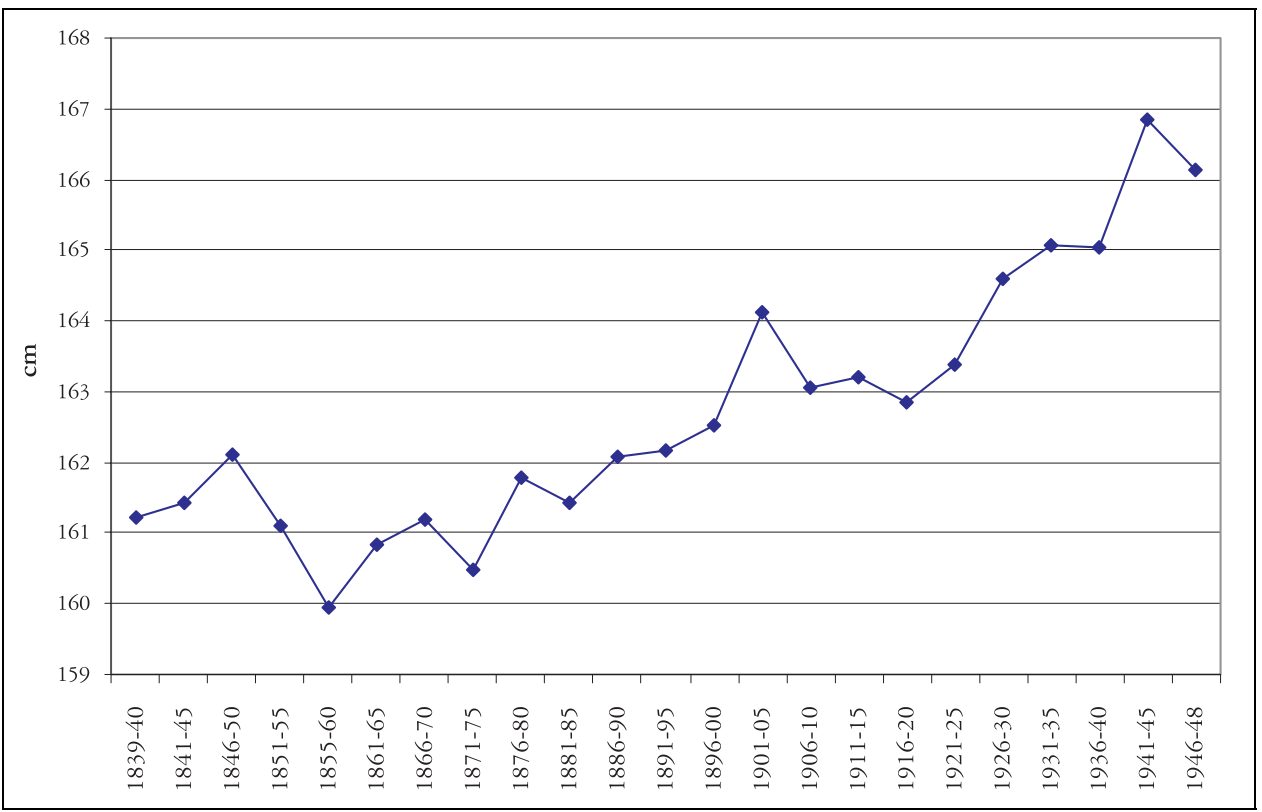

\section{II.1. Talla, pobreza y stunting a mediados del siglo XIX}

La historiografía reciente de historia antropométrica ha venido señalando la existencia de tiempos duros para el bienestar de las gentes en las décadas centrales del siglo XIX. De acuerdo con ella, algunas regiones y en particular algunas provincias registraron un deterioro de la altura media de los mozos que pudo manifestarse en pérdidas significativas en algunos casos$^{9}$. Los resultados obtenidos en este trabajo revelan también un deterioro de la talla en las décadas centrales de dicha centuria. Las series sugieren una caída de la altura de los mozos castellanos y leoneses para las generaciones nacidas desde 1845 a 1875. En ese lapso de tiempo la talla media de los jóvenes de la región menguó en 1,3 centímetros, coincidiendo con una caída de los salarios reales, el impacto de las crisis de subsistencias y, por consiguiente, con menores posibilidades alimenticias ${ }^{10}$. El dete-

9 MARTínez CARrión, José Miguel, «Estatura, salud y bienestar en las primeras etapas del crecimiento económico español. Una perspectiva comparada de los niveles de vida», Documento de trabajo de la AHE, 2001, 0102; MARTÍNEZ CARRIÓN, José Miguel, "Calidad de vida y bienestar biológico..., págs. 673-706.

10 Moreno LÁZARo, Javier, «¿Fomentó el capitalismo agrario la desigualdad? Salarios y niveles de vida en Castilla la Vieja, 1751-1861», en: MARTínEZ CARRIÓN, José Miguel (ed.), El nivel 
rioro que la talla media sufrió en el norte de Castilla y León en las décadas centrales de siglo se ha visto con desigual intensidad en otras regiones españolas y en las áreas europeas más industrializadas ${ }^{11}$. En algunos países del Hemisferio occidental, casos de Gran Bretaña, Alemania, Holanda, Estados Unidos, el deterioro de los promedios de altura alcanzó mayores proporciones de las que se conocen en España.

El detrimento del bienestar biológico humano se sugiere por el porcentaje de cortos de talla, o mozos que no alcanzaron la talla mínima de $156 \mathrm{~cm}$ exigida para el sorteo y el posterior destino militar. Este procedimiento fue realizado tempranamente por don Laureano Figuerola ${ }^{12}$. De acuerdo con éste, el deterioro de la talla en las décadas centrales del XIX se observa en el caso estudiado, y aún en las siguientes, sobre todo a la altura del quinquenio de 1890-94 (de los nacidos en 1871-75), en que el número de mozos con talla por debajo de 156 $\mathrm{cm}$ aumentó hasta el 27,3 por 100 entre los mozos castellanos y leoneses (Tabla 4 y Figura 3). Las provincias incluidas en esta muestra lideraban a mediados del XIX el ranking nacional en porcentaje de cortos sobre el total de reclutas en otros estudios realizados ${ }^{13}$. Aunque parte de este incremento cabe imputárselo al cambio de edad reglamentaria, que pasó de 20 a 19 años en el reemplazo extraordinario del verano de 1885 , los incrementos observados antes de ese año revelan un aumento de la malnutrición, relacionada probablemente por la sinergia producida entre las infecciones, el trabajo infantil y la pobreza de las dietas, escasas en nutrientes y proteínas.

El incremento del fenómeno enunciado, rozando casi el enanismo, en sólo una década fue asombroso, pasando del 13 al 23,7 por 100 entre los reempla-

de vida en la España rural..., págs. 75-112; MORENO LÁZARO, Javier, «El nivel de vida en la España atrasada entre 1800 y 1936. El caso de Palencia», Investigaciones de Historia Económica, 4 (2006), págs. 9-21.

11 Martínez Carrión, José Miguel, «Estatura, salud y bienestar en las primeras etapas del crecimiento económico español. Una perspectiva comparada de los niveles de vida», Documento de trabajo de la AHE, 2001, 0102; KOMLOS, John, «Srinking in a Growing Economy? The mystery of physical stature during the Industrial Revolution», Journal of Economic History, 58/3 (1998), págs. 779-802; KOMLOS, John (ed.), The biological standard of living on three continental. Further explorations in anthropometric history, Boulder et al., 1995; KOMLOS, John y BATEN, Joerg (eds.), The Biological Standard of Living in Comparative Perspective, Franz Steiner, Stuttgart, 1998; STECKEL, Richard H. y FLOUD, Roderick (eds.), Health and Welfare during Industrialization, Chicago, Chicago University Press, 1997.

12 Figuerola, Laureano, «La talla de los mozos para el servicio militar sorteados y medidos en las quintas de 1858 a 1867», Memorias de la Real Academia de Ciencias Políticas y Morales, tomo VII, 1893, págs. 305-311.

13 Martínez CARrión, José Miguel, «Estatura, salud y bienestar en las primeras etapas del crecimiento económico español. Una perspectiva comparada de los niveles de vida», Documento de trabajo de la AHE, 2001, 0102; FEIJOO-GÓMEZ, A., Quintas y protesta social en el siglo XIX, Madrid, Ministerio de Defensa, 1996; Gomez MendozA, Antonio y Perez MoredA, Vicente, «Estatura y nivel de vida en la España del primer tercio del siglo XX», Moneda y Crédito, 174 (1985), págs. 29-64. 
zos de 1866-70 a 1875-80, compuestos por mozos de 20 años de edad. Los aumentos porcentuales de esos años evidencian los efectos negativos de las crisis de subsistencias y epidémicas producidas en el entorno de las cohortes nacidas en 1851-60 y que, siendo adolescentes, también vivieron las crisis de 186768 , en plena fase del estirón y maduración física. El embate de las crisis económicas y demográficas en los años de la infancia, en los primeros años de vida, y del estirón puberal, entre los 13 y 18 años, segó una buena parte del crecimiento físico potencial que podría haberse registrado en circunstancias normales para dichos reemplazos.

FIGURA 3. EVOLUCIÓN DE LOS MOZOS CON TALLA INFERIOR A 156 CM, REEMPLAZOS DE 1856 A 1966

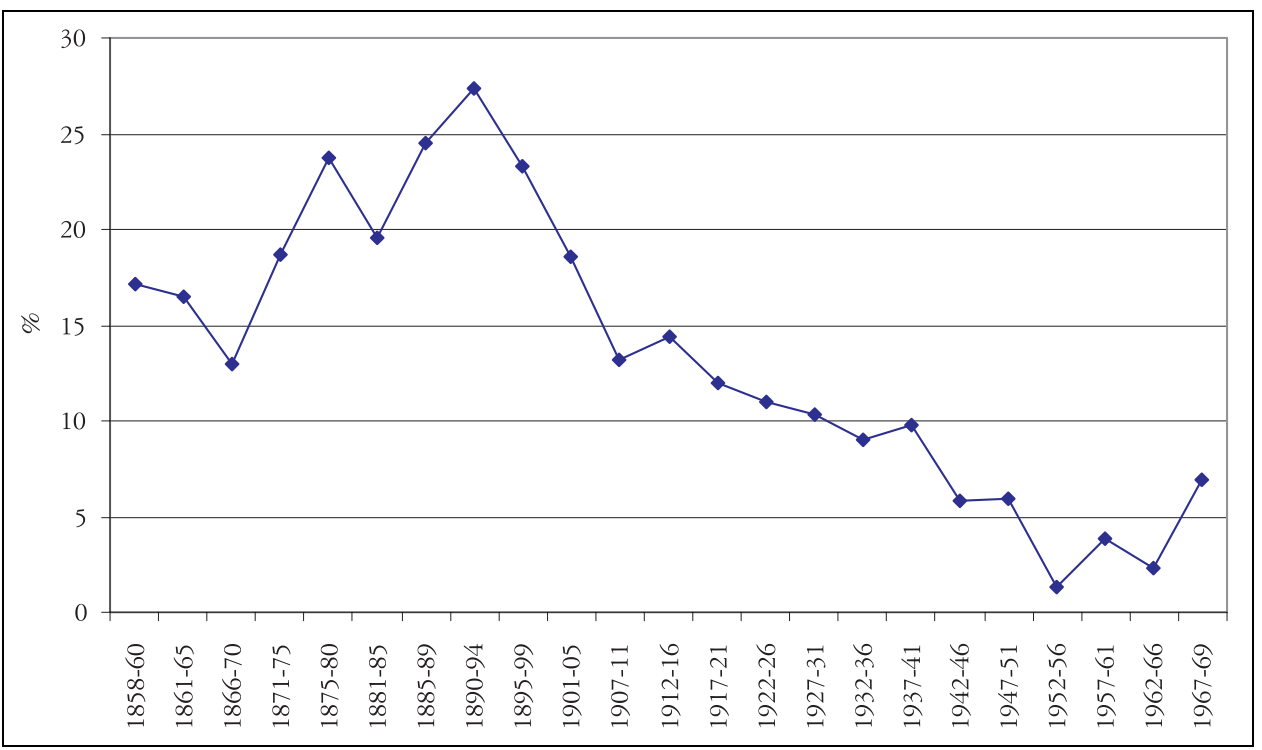

En un contexto en que se incrementaron los riesgos de mortalidad, debido a la prevalencia de enfermedades epidémicas y al impacto de las crisis de subsistencias, las infecciones pudieron deteriorar las condiciones fisiológicas de unos cuerpos ya bastante canijos, debilitados y empobrecidos, extendiendo la morbilidad e incrementando la intensidad de la malnutrición que pudo ser severa en algunos sectores sociales. Este fenómeno de prevalencia de estaturas bajas a edades determinadas y sobre todo al final del crecimiento físico ha sido denominado en la literatura biomédica y antropométrica como stunting, un fenómeno que sin llegar a rozar situaciones de enanismo, o rozándolo en extremo, mostraría la existencia de tallas determinadas por la escasez de recursos 
y unas dietas con insuficientes aportes de energía y proteínas ${ }^{14}$. En otras palabras, se recrudecieron los niveles de pauperismo y miseria fisiológica y empeoraron las condiciones del bienestar humano.

FIGURA 4. NiVELES DE PAUPERISMO A PARTIR DE LOS MOZOS EXCLUIDOS POR RAZONES SOCIALES, REEMPLAZOS DE 1858 A 1966

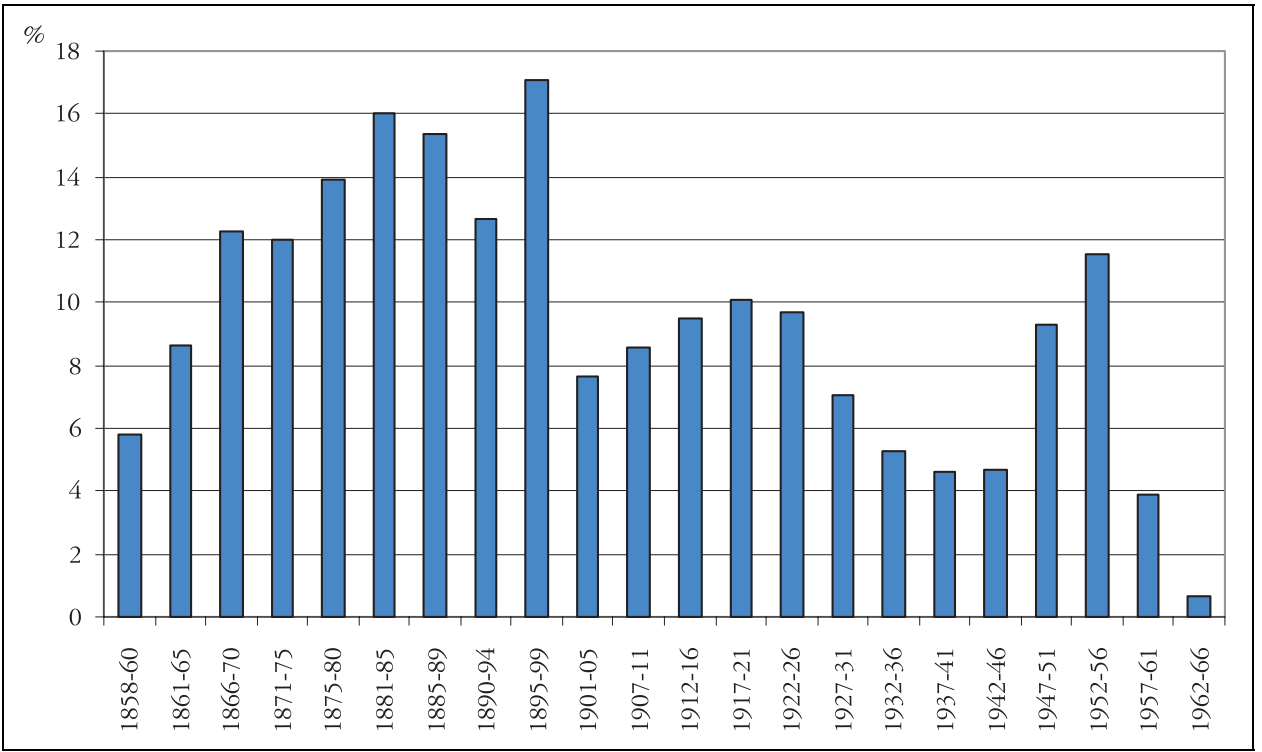

La acentuación de los niveles de pobreza en este periodo del siglo XIX se puede observar también a través de los mozos excluidos por razones sociales. Los jóvenes cuyos ingresos eran imprescindibles para el sostenimiento de su familia, bien por ser huérfanos de padre, tener otro hermano en el servicio militar o hallarse inactivo su progenitor, eran exonerados de sus obligaciones militares. Los cálculos presentados en la Figura 4 evidencian ese deterioro socioeconómico progresivo sufrido por los menesterosos en Castilla y León hasta 1885. Así, del 5,8 por 100 excluidos por razones sociales en los reemplazos de 1858-60 se pasó al 16 por 100 en los de 1881-85. Con todo, el porcentaje real de jóvenes

14 Sommer, A. y Lowentein, M.S., "Nutritional status and mortality: A prospective validation of the QUAC stick», American Journal of Clinical Nutrition, 28 (1975), págs. 287-292; MARTORELL, R., "Child growth retardation: A discussion of its causes and its relationship to heath», en: BlaXter, K. y WATERLOW, J.C. (eds. ), Nutritional adaptation in man, Londres, John Libbey, 1985, págs. 13-29; FOGEL, Robert W., The escape from bunger and premature death, 1700-2100. Europe, America and the Third World, Cambridge, Cambridge University Press, 2004. 
para quienes la realización del servicio militar habría supuesto una caída insostenible de los ingresos de sus familias fue muy superior, ya que, por lo común, los cortos no alegaban su situación de pobres, pues contaban con la exclusión total de forma segura. En otras palabras, el agregado resultante de la suma de los cortos de talla y los excluidos por razones sociales mide de manera mucho más certera el alcance real de la depauperación que sufrieron las clases populares en la Castilla y León del siglo XIX. Los resultados de ambos ejercicios nos revelan el incremento de la malnutrición, la extensión de la pobreza y la amplitud del deterioro del bienestar físico y del nivel de vida biológico.

\section{II.2. La mejora del nivel de vida biológico desde finales del siglo XIX}

La coyuntura finisecular supuso un punto de inflexión en la tendencia del bienestar biológico. El pesimismo reinante entre las generaciones nacidas a mediados del siglo XIX dio paso a un optimismo relativo si se tiene en cuenta el punto de partida. Los mozos nacidos desde la década de 1880 mostraron unos niveles de vida relativamente mejores. Así lo revela el aumento progresivo de sus promedios de talla y la disminución del porcentaje de cortos o apartados del sorteo por su condición de pobres. El número de mozos cortos de talla cayó drásticamente, pasó del 27,3 por 100 en los reemplazos de $1871-75$ al 9 por 100 entre los de 1911-15 (Figuras 1, 2, 3 y 4). Sólo el descenso de los salarios reales y la agudización de los problemas de subsistencias durante los años de la I Guerra Mundial quebraron momentáneamente esta mejora de los niveles de vida biológicos. De hecho, se observa un deterioro del pauperismo por el incremento de mozos excluidos por razones sociales entre 1905 y 1921, tras un periodo anterior marcado por el descenso de dichos niveles. El crecimiento de la estatura física cifrado en más de $3 \mathrm{~cm}$ entre las cohortes de 1871-75 y 191115 es imputable al incremento de los salarios, más moderado que el que experimentó la talla, y sobre todo a las mejoras sanitarias, laborales y residenciales experimentadas, en especial desde 1900 en adelante.

\section{II.3. El bienestar humano desde la Guerra Civil y el Franquismo}

Las condiciones del bienestar humano y el nivel de vida durante el Franquismo, y más en concreto desde la Guerra Civil hasta comienzos de los años 50 , mediando entre ambas fechas la larga posguerra, son objeto de controversia entre los especialistas desde hace años. Los resultados han mostrado que la autarquía franquista fue desastrosa en términos de política económica y que los indicadores que miden la riqueza y la renta de los españoles tendieron a la baja. Salvo las principales magnitudes demográficas, los índices de bienestar material y del nivel de vida en general disminuyeron. La renta per capita, los salarios 
reales y el consumo cayeron en picado; tras los años inmediatos de la posguerra, los años 1944-48 fueron los más duros en la mayor parte del país y afectaron sobre todo a las clases populares. Los estándares de vida alcanzados en 1935 no se recuperaron hasta aproximadamente $1955^{15}$. También recientes estudios antropométricos han mostrado un deterioro relativo de la altura media para los reemplazos que vivieron la posguerra y la etapa del primer franquismo ${ }^{16}$; paralelamente, estudios sobre la nutrición revelan una caída del consumo de energía y proteínas entre los años de la II República y comienzos de los años $50^{17}$. Aunque la tasa bruta de mortalidad disminuyó en la década de 1940 por la difusión de nuevas tecnologías médicas, como las sulfamidas y los antibióticos, hubo muchas zonas - principalmente rurales - donde los progresos médicos y sanitarios apenas se observaron, incluso se deterioraron por falta de infraestructura y equipamiento ${ }^{18}$. Por todo ello se ha calificado la etapa del primer franquismo como desastrosa desde el campo del bienestar, la salud y la nutrición, que manifestaron un notable retroceso.

¿Qué ocurrió en las poblaciones analizadas? A juzgar por los datos representados en la Figura 1, los niveles de vida biológicos de la población castellana y leonesa estudiada mejoraron en términos relativos durante la Autarquía. Pero en los años de franquismo conviene tener en cuenta una serie de prevenciones. La primera de ellas tiene que ver con la prolongación de la serie de altura, sólo hasta 1948 según el año del nacimiento del mozo (por cohortes), aunque suficiente para una evaluación de los años autárquicos más duros. La segunda, más significativa, es que la muestra es pequeña y está compuesta mayoritariamente por núcleos rurales, donde, sea por las mayores posibilidades de aprovisionamiento de alimentos en el mercado negro o por las de obtención de ingresos alternativos, pudieron disfrutar de niveles de vida relativamente mayores que en los núcleos urbanos. Los problemas de abastecimiento y consumo alimenticio fueron mayores en las ciudades.

Los datos de la muestra constatan un aumento de la talla de $3 \mathrm{~cm}$ entre las cohortes de 1911-15 y 1946-48, cuando en otras regiones españolas no sucedió

15 Barciela, Carlos (ed.), Autarquía y mercado negro. El fracaso económico del primer franquismo, 1939-1959, Barcelona, Crítica, 2003; Prados DE LA Escosura, Leandro, El progreso económico de España (1850-2000), Madrid, Fundación BBVA, 2003.

16 Coll, Sebastian, Quiroga, G., Height and the standard of living in 20th century Spain: A preliminary report, Documentos de Trabajo 94.05, Departamento de Economía, Universidad de Cantabria, 1994; MARTínez Carrión, José Miguel, «Niveles de vida y desarrollo económico en la España contemporánea. Una visión antropométrica», Revista de Historia Económica, 3 (1994), págs. 685-716; MARTíneZ CARRIón, José Miguel, «Estaturas, desigualdad regional y desarrollo económico..., págs. 206-228; MARTínez CARrión, José Miguel, «Calidad de vida y bienestar biológico..., págs. 673-706. QUIROGA, Gloria: «Estatura, diferencias regionales..., págs.175-200.

17 Cussó, Xavier, «El estado nutritivo de la población española, 1900-70. Análisis de las necesidades y disponibilidades de nutrientes», Historia Agraria, 36 (2005), págs. 329-358.

18 Bernabeu-Mestre, Josep, Caballero-Pérez, P., Galiana-SÁnChez, M.E. y Nolasco, A., «Niveles de vida y salud en la España del primer franquismo: las desigualdades en la mortalidad infantil», Revista de Demografía Histórica, 24/1 (2006), págs. 181-202. 
lo mismo. Si acortamos, y tomamos como referencia el periodo de los nacidos entre 1916 y 1945, los promedios de talla sugieren un avance de casi $4 \mathrm{~cm}$. En otros países el aumento fue incluso mayor, pese a los problemas ocasionados por la II Guerra Mundial. Ahora bien, un análisis más fino de los datos documenta la existencia de algunos problemas y de carencias que terminaron por afectar al bienestar humano durante la primera etapa franquista. Aunque se advierte una mejora relativa respecto de otras áreas españolas, podemos observar una leve caída de la talla en los reemplazos de 1837-41. La talla media alcanzada en los reemplazos de 1922-26, la más alta del primer tercio del siglo XX, no se recuperó hasta 1947-51. Además, los promedios de talla de 195256 se estancaron en el quinquenio siguiente de 1957-61.

El hecho puede ser revelador de las dificultades y las carencias de energía y proteínas que arrastraron los castellanos en los «años del hambre». Dos indicadores así lo manifiestan. De un lado, el porcentaje de cortos de talla aumentó algo entre los reclutas de 1937-1941 y 1947-51 (Figura 3). De un porcentaje muy bajo alcanzado en las cohortes nacidas durante la II República (1931-35), cifrado en 1,3 por 100, se pasó al 6,9 por 100 en las cohortes de 1946-48, habiéndose multiplicado por cuatro la tasa de 'enanismo'. De otro, casi paralelamente, aumentaron los niveles de pauperismo estimados por exclusiones de diversa razón social. La tasa de excluidos por tales motivos se duplicó entre 1942-46 y 1952-1956. Los resultados invitan a repensar la visión ligeramente optimista que a primera vista ofrecen los promedios de talla pues con otros campos de los datos antropométricos, como aumento del stunting y la pauperización sugieren que las carencias nutricionales también pudieron afectar a la zona «nacional», precisamente la de mayor reserva de recursos trigueros y aumentaron con ello los problemas sociales y económicos de buena parte de familias y los hogares castellano-leoneses.

\section{II.4. Comparaciones de talla según área de residencia y rural-urbana}

Los datos antropométricos del norte de Castilla y León se comparan con la principal serie histórica disponible, la del sudeste de España ( $\mathrm{N}=186387$ mozos observados con talla entre las generaciones nacidas entre 1837 y 1948), otra gran área española con una fuerte economía de base agraria y algunos núcleos industriales, como Elche y Cartagena ${ }^{19}$. Los resultados mostrados en la

19 Martínez Carrión, José Miguel y Pérez Castejón, Juan José, «Creciendo con desigualdad. Niveles de vida biológicos en la España rural mediterránea desde 1840», en: Martínez Carrión, J.M. (ed), El nivel de vida en la España rural..., págs. 405-460. Para el caso de Cartagena, ver MARTíneZ CARRIÓn, José Miguel, «Salud, ambiente y bienestar biológico: la estatura en el municipio de Cartagena (siglo XIX)», Áreas. Revista Internacional de Ciencias Sociales, 24 (2004), págs. 157-190. Sobre la evolución de las tallas en Elche, véase MARTínEZ CARRIÓN, José 
Figura 5 revelan que la caída de la talla para los nacidos en Castilla y León durante las décadas centrales del XIX fue mucho más aguda que en el sudeste español y que las mejoras obtenidas en el bienestar biológico, desde 1870 hasta la I Guerra Mundial, fueron menos significativas. A lo largo del período, la ventaja en favor de sudeste de España desvela la posición rezagada que, en términos de bienestar, padeció Castilla y León a las puertas de la Gran Guerra europea. Sin embargo, desde entonces, los nacidos castellano-leoneses tendieron a equiparse casi con la media de altura alcanzada en el sudeste de España.

Figura 5. TALla MEdia EN EL NORTE DE CASTILla Y LEÓN Y SUDESTE DE ESPAÑA, SEGÚN COHORTE DE NACIMIENTO Y EDAD DE RECLUTAMIENTO

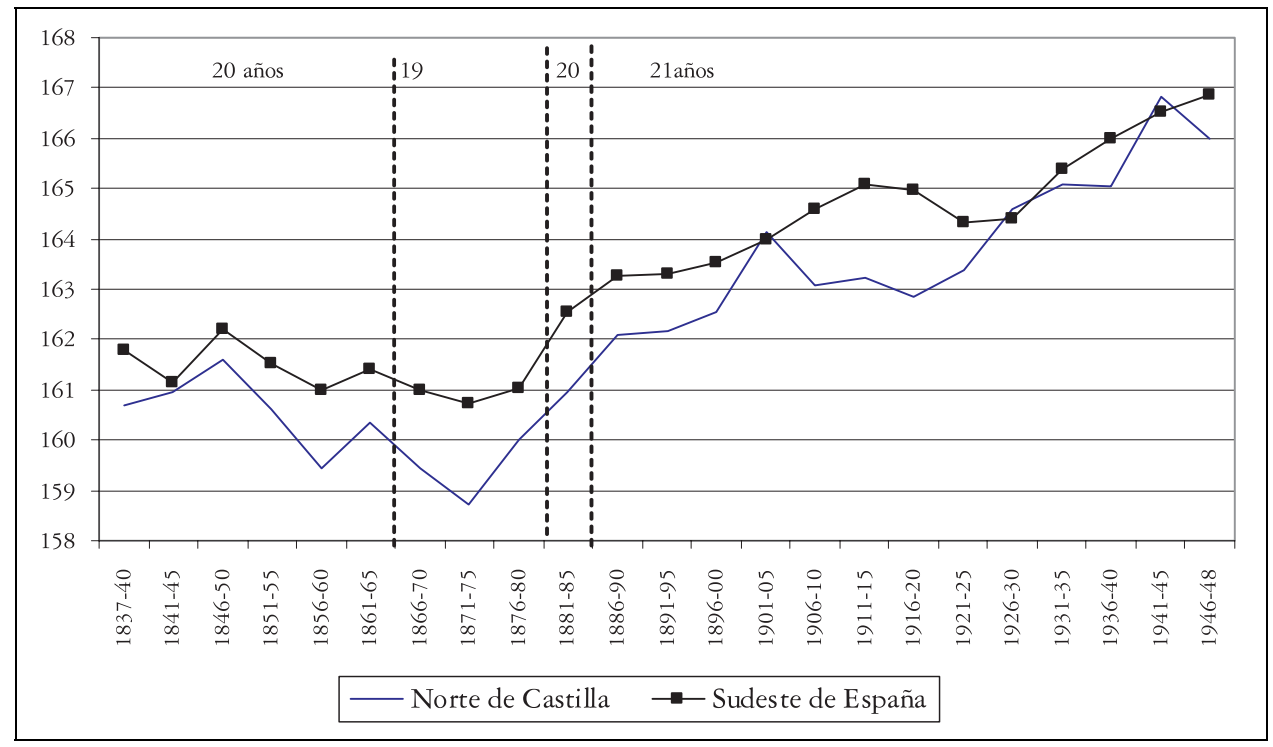

La convergencia producida en el bienestar humano al final del periodo analizado entre ambas series se refuerza en el ámbito rural. Así lo afirman los datos de estaturas medias según su residencia rural. La Figura 6 revela que los habitantes del sudeste de España sufrieron más penalidades en los años de la postguerra que los castellanos. Los datos pueden tener interés para el estudio de los costes regionales de la política económica y agraria del primer franquismo.

Las disparidades territoriales en el nivel de vida a escala regional se muestran también significativas en la comparación entre los ámbitos rural y urbano, aún señalada la prevención con la que hay que emplear estas categorías en Cas-

Miguel y PÉREZ CASTEJón, Juan José, «Height and standard of living during the industrialisation of Spain: The Case of Elche», European Review of Economic History, II/2 (1998), págs. 201-230. 
tilla y León. La Figura 7 evidencia que el deterioro del bienestar a lo largo de las décadas centrales del siglo XIX fue mucho más perceptible en el mundo urbano que en el rural, donde incluso se asistió a una substancial mejora de los niveles a lo largo de la primera mitad de los reemplazos de la década de 1880 , la de los nacidos en los años 1860, inexistente en las capitales.

Figura 6. TALLA MEDia EN LAS ÁREAS RURALES DE CASTILla Y LEÓN Y SUDESTE DE ESPAÑA, COHORTES 1839-1948

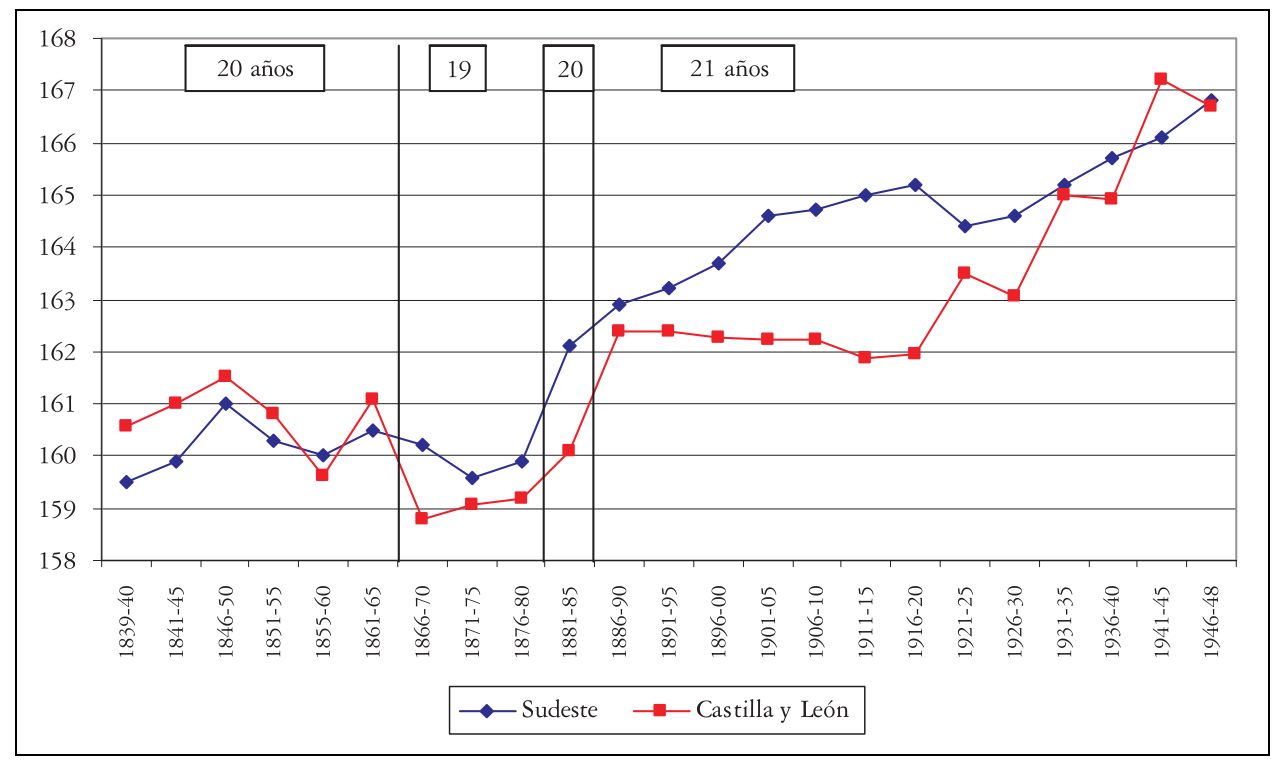

Los datos sugieren una posible "penalización urbana» del bienestar en las primeras fases de la industrialización. Ahora bien, las desventajas de la vida urbana no fueron consecuencia del coste social que supuso la implantación de la economía de fábrica, sino de la continuidad de las formas de producción preindustrial ${ }^{20}$. La supervivencia de la manufactura textil a escala doméstica — todavía en 1850 era la actividad más extendida en las capitales castellanas y leonesas, pese al acoso de la pujante y competitiva industria lanera catalana- exigió una sobreexplotación de la fuerza de trabajo y terminó afectando al bienestar de los trabajadores deteriorándolo de forma muy apreciable. Multitud de testimonios emanados de la propia Administración y de la Iglesia corroboran el empleo de niños que trabajaban hasta 14 horas diarias en insalubres talleres textiles, caren-

20 Martínez Carrión, José Miguel y Moreno LÁZaro, Javier, «Was There an Urban Height Penalty in Spain, 1840-1913?», Economic and Human Biology, 5/1 (2007), págs. 144-164. 
tes de iluminación y expuestos a las 'miasmas' y al contagio del carbunco, enfermedad ocasionada por las materias con las que eran cardadas las lanas. Las familias de tejedores, curtidores y jornaleros del campo residían hacinados en infectas «casas-habitaciones» sin hogar ni ventanales. Algunos informes oficiales de la época revelan el encanijamiento de los jóvenes palentinos: «¿̇(...) habrá ya quién torture su imaginación investigando misteriosas causas que expliquen el porqué pululan tantos jóvenes encanijados por nuestros hospitales y casas de asilo?» ${ }^{21}$.

Figura 7. DifERENCIAS URBANO-RURALES DE TALLA EN CASTILla Y LEÓN POR COHORTES

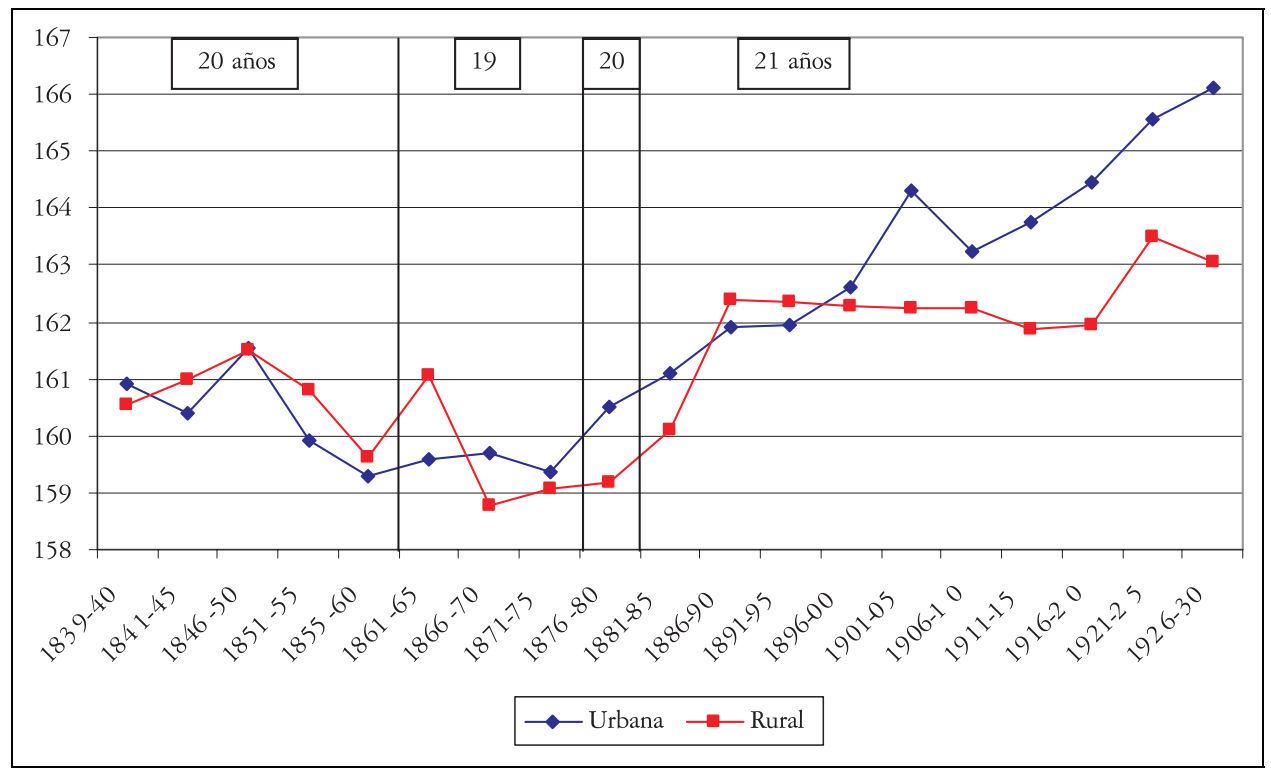

Entre tanto, las condiciones ambientales de trabajo en el mundo rural eran relativamente más benévolas que en mundo urbano de esa época. Un informe médico del término municipal de Astudillo resulta extraordinariamente revelador, al interrogarse sobre las causas de la 'miseria fisiológica y la vejez anticipada' que padecían los operarios de las fábricas de tejidos frente a los jornaleros del campo. Dicho informe señala que ambos colectivos profesionales compartían 'una alimentación insuficiente con pocos principios proteicos' y residencia en 'casas pequeñas donde escasea la luz y el aire'. Pero encuentra algunas diferencias

21 Boletín Oficial de la Provincia de Palencia, 20 de octubre de 1884. 
medioambientales: mientras la jornada laboral de los tejedores transcurría 'respirando un aire cargado de impurezas, llamando la atención el desaseo por el sucio material que manejan', que explicaría las condiciones de su miseria fisiológica, los braceros del campo eran 'fuertes y robustos la mayor parte de ellos, pues sin embargo de habitar idénticas viviendas y tener igual alimentación que los de la clase industrial, respiran un aire oxigenado y puro durante todo el día que dura el trabajo'22. De acuerdo con esta información, existiría un ambiente urbano que penalizaría el bienestar biológico, dadas las condiciones de higiene, trabajo y vivienda, frente a un ambiente rural relativamente más saludable.

Sin embargo, algunas zonas rurales comenzaron a perder las ventajas relativas de disfrutar de un medio ambiente más sano. Así al menos se desprende de las series de reemplazos de los mozos llamados a filas desde finales de la década de 1880. En adelante, las ganancias relativas de bienestar en las capitales castellanas podrían explicarse por la mejora en las condiciones de trabajo de los operarios no cualificados y la paulatina desaparición de los inhóspitos obradores textiles. Los avances sanitarios, las mejoras en el hábitat, el acceso al agua potable en los hogares y el adecentamiento de las calles no llegaron a los pueblos hasta los años del franquismo, lo cual ensanchó la brecha entre el campo y la ciudad en términos de calidad de vida, y sobre todo de nivel de vida biológico, desde comienzos del siglo XX. De hecho, la talla de los quintos nacidos en las zonas rurales se estancó entre las cohortes de 1885 y 1917, en torno a los 162 $\mathrm{cm}$. Y aunque hubo mejoras en las tallas de los nacidos durante las décadas de 1920 y 1930, las diferencias a favor del mundo urbano siguieron siendo importantes. Estas desaparecieron hacia finales del periodo. Sobresale el hecho al coincidir este avance en los reemplazos de las décadas de 1950 y 1960, al comienzo de la modernización del campo. De acuerdo con los datos de la Figura 6 , al final del periodo los avances en las tallas medias rurales en tierras de Castilla y León fueron de tal magnitud que superaron incluso a las tallas medias de los mozos del sureste español. El fenómeno migratorio podría explicar en parte estas variaciones.

\section{II.5. Las desigualdades sociales: diferencias de talla según la profesión}

El siguiente ejercicio muestra las diferencias de talla de los reemplazos según la profesión. La Figura 8 muestra la evolución del grupo más numeroso, el de los trabajadores del campo (compuesto por ‘jornaleros' y 'braceros'), que a tenor de los datos sufrieron más que el resto de la población asalariada el deterioro de los niveles de vida experimentado en las décadas centrales del XIX. Superada esta coyuntura tan dañina para los intereses de los menesterosos, los niveles de vida de los jornaleros se equipararon a los de los trabajadores a jornal

22 Archivo Municipal de Astudillo (AMA), Caja 168, Expediente 4. 
no vinculados a la actividad agraria. Sin embargo, las disparidades se agudizaron desde 1900. Querría ello decirse que las mejoras experimentadas en la distribución de la renta no llegaron a los trabajadores del campo. Los datos sugieren una segunda conclusión de gran interés en la interpretación de los bajos niveles de productividad del trabajo en el agro castellano y leonés: quienes persistieron en las tareas agrarias tuvieron, progresivamente y en términos relativos, una menor talla física.

Figura 8. TALLA MEDIA DE LOS TRABAJADORES DEL CAMPO, COHORTES1850-1925

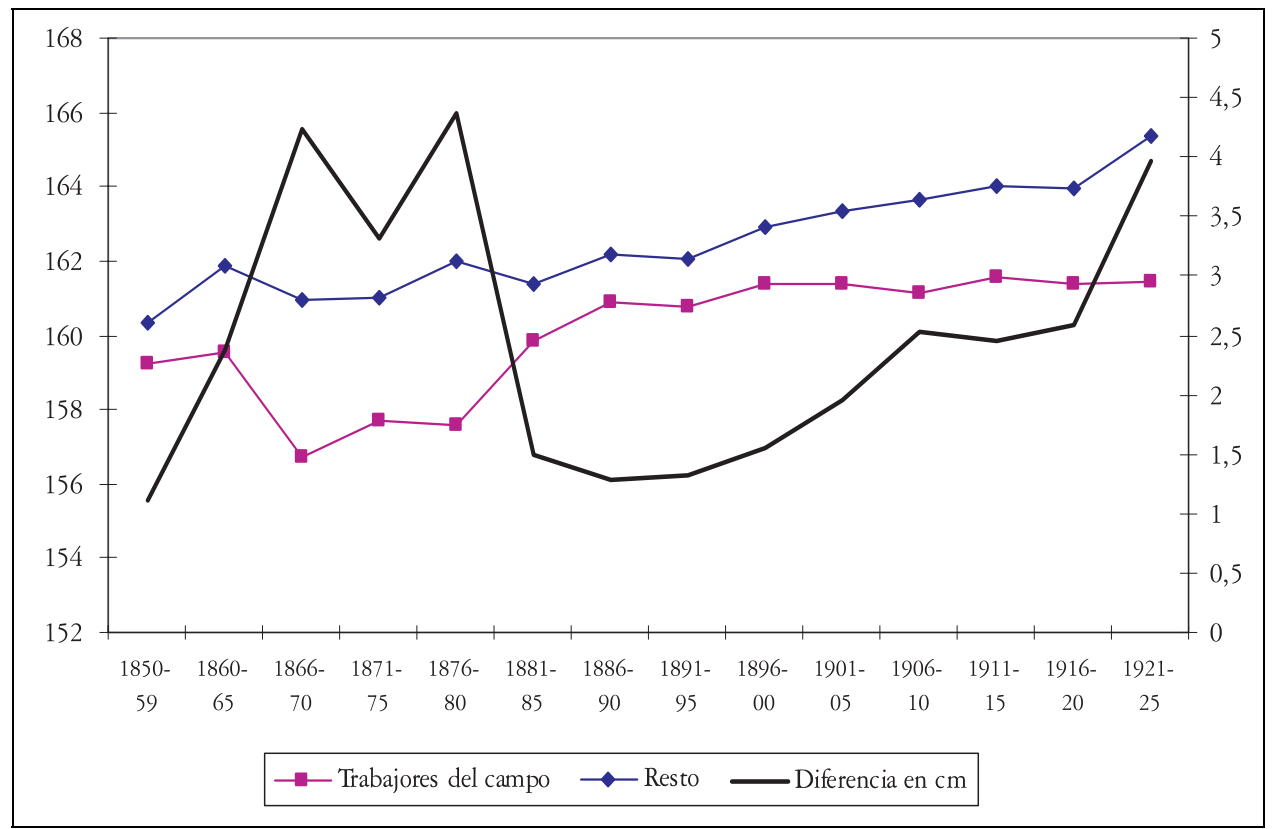

Los propietarios de la tierra atravesaron por las mismas penalidades. La equiparación de sus niveles de vida hasta comienzos de la década de 1920 evidenciaría las dificultades económicas sufridas desde los años finiseculares por los denominados «propietarios muy pobres» (Figura 9). La situación mudó sustancialmente desde entonces, presumiblemente merced a la política de precios agrarios aplicada tras la promulgación de la Ley de Subsistencias de 1915. 
FIGURA 9. TALLA MEDIA DE LOS ACTIVOS CASTELLANO-LEONESES: ASALARIADOS DEL CAMPO Y PROPIETARIOS AGRÍCOLAS, COHORTES 1850-1925

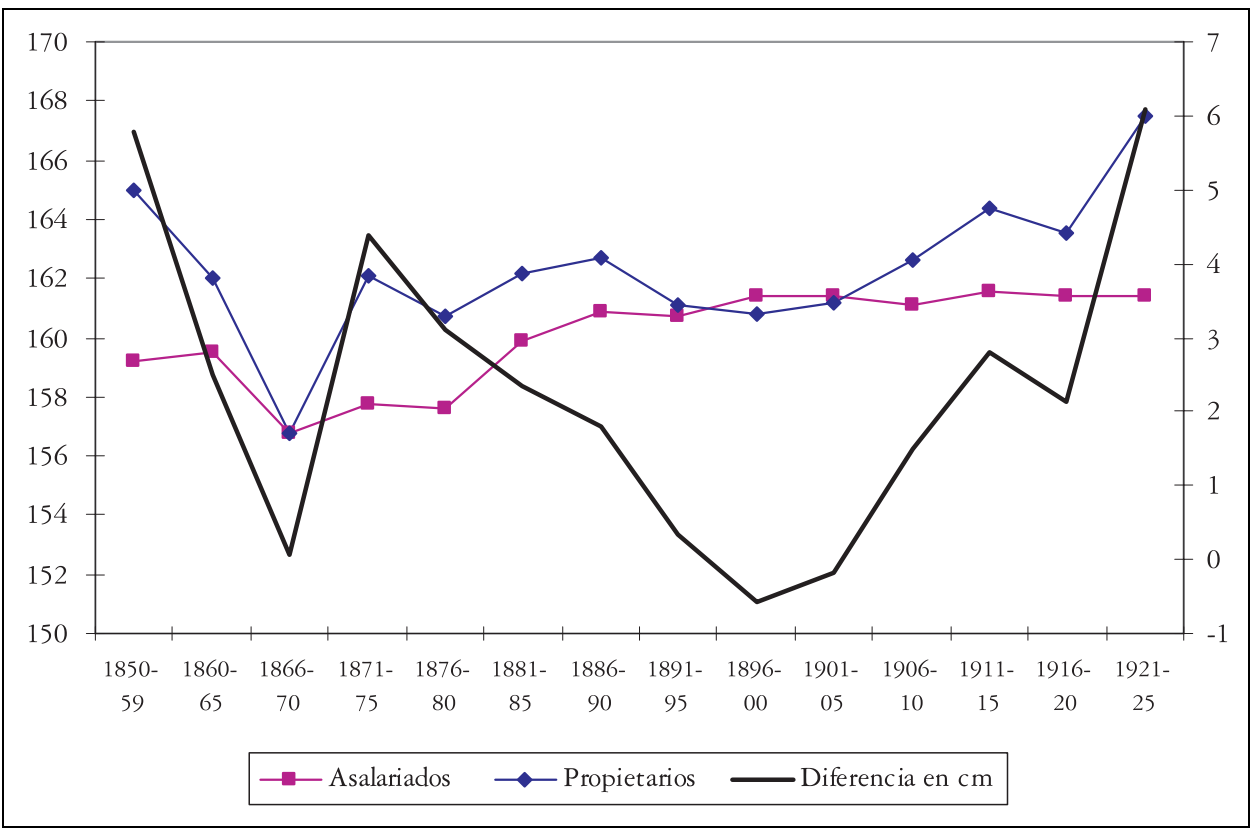

Las diferencias de talla observadas entre trabajadores del campo y asalariados en el sector secundario son muy similares. La Figura 10 revela que los jornaleros del campo no disfrutaron, al menos con la misma intensidad, de las mejoras salariales ni de las condiciones de trabajo obtenidas desde los años de la dictadura de Primo de Rivera por los trabajadores en el sector industrial. La evolución de las tallas difiere significativamente a favor de los últimos, cuya estatura media aumentó entre 1 y $3 \mathrm{~cm}$ desde las generaciones de 1906-11.

TABla 5. TALLA MEDIA (CM) DE LOS OPERARIOS DE FÁBRICAS, COHORTES 1840-1914

\begin{tabular}{|c|c|c|c|c|c|c|}
\hline Período & $\begin{array}{c}\text { Cuero y } \\
\text { calzado }\end{array}$ & $\begin{array}{c}\text { Materiales de } \\
\text { Construcción }\end{array}$ & Textil & Artes gráficas & Metal & Alimentación \\
\hline $1840-1865$ & 160,8 & & 159,3 & & & 160,0 \\
\hline $1866-1880$ & 158,3 & 154,4 & 157,2 & 160,6 & 161,1 & 162,2 \\
\hline $1886-1893$ & 160,9 & 160,3 & 158,6 & 161,2 & 161,3 & 161,7 \\
\hline $1894-1914$ & 160,0 & 160,1 & 160,7 & 161,4 & 162,6 & 161,8 \\
\hline
\end{tabular}


TABla 6. TAlla Media (CM) DE lOS ARTESANOS, COHORTES 1840-1914

\begin{tabular}{|c|c|c|c|c|c|c|}
\hline Período & Sastres & Albañiles & Herreros & Carpinteros & Pintores & Zapateros \\
\hline $1840-1865$ & & 160,8 & 158,6 & 161,8 & & 161,7 \\
\hline $1866-1880$ & 157,8 & 161,1 & 159,6 & 160,3 & & 158,1 \\
\hline $1886-1893$ & 158,3 & 160,4 & 159,9 & 162,1 & 161,5 & 160,9 \\
\hline $1894-1914$ & 162,7 & 162,1 & 161,6 & 162,6 & 162,6 & 159,7 \\
\hline
\end{tabular}

Los activos industriales no constituyeron un colectivo homogéneo. Los datos reproducidos en las Tablas 5 y 6 evidencian la dureza de los trabajos en las fábricas de tejidos y de curtidos, así como en las canteras y fábricas de ladrillos y tejas, y unas condiciones de vida laboral que obstaculizaron el crecimiento físico de los empleados en ellas. Los operarios de las fundiciones y los trabajadores de la madera compondrían la «aristocracia obrera», por emplear la terminología al uso.

Figura 10. TALla MEdia DE los TRABAJAdORES A JORNAL: TRABAJADORES DEL CAMPO, ARTESANOS Y OPERARIOS FABRILES, COHORTES 1850-1925

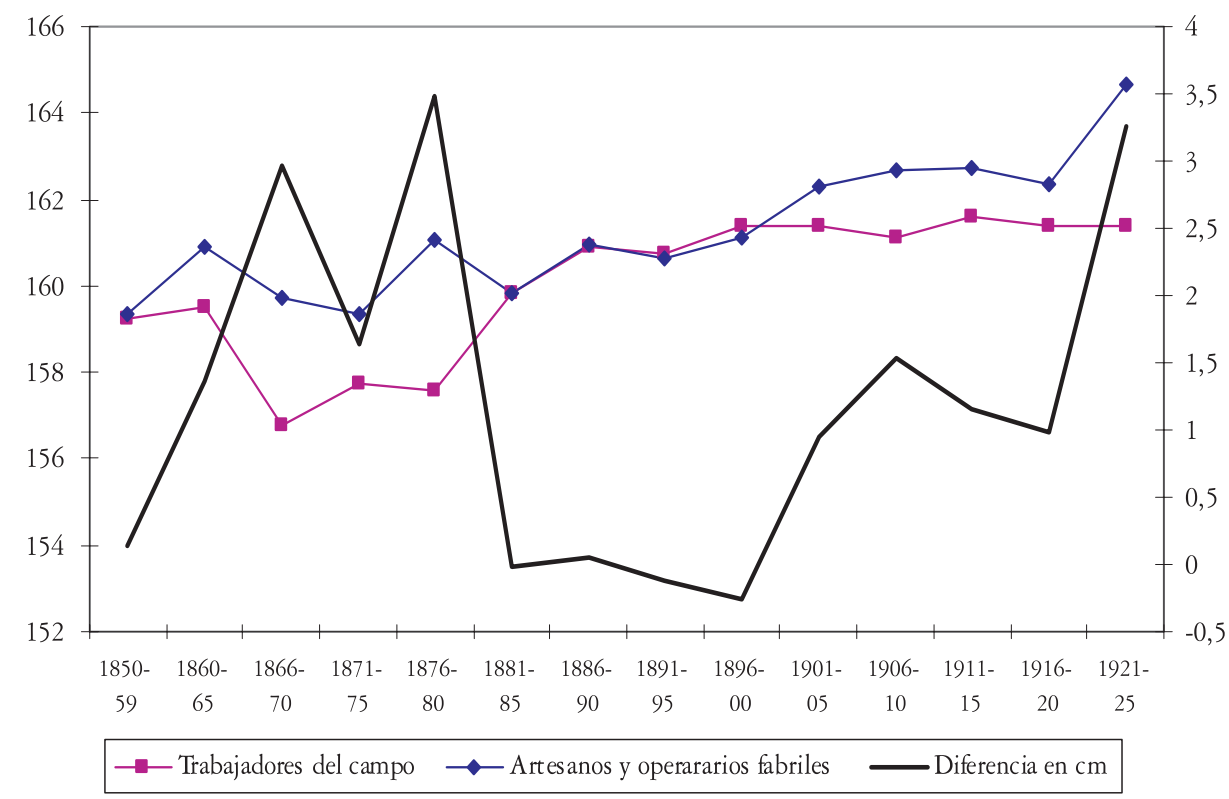




\section{CONCLUSIONES}

La tendencia secular de la estatura en Castilla y León muestra un balance del bienestar biológico bastante pobre en términos relativos hasta las generaciones nacidas a mediados del siglo XX. Los datos revelan la existencia de un ciclo de deterioro del nivel de vida en las décadas centrales del siglo XIX. El declive de la talla no sólo fue más acusado en Castilla y León que en el sureste de España entre los nacidos de 1850 a 1870, sino que los castellanos mantuvieron bajos sus niveles de bienestar humano hasta las cohortes de 1920. El incremento de la talla entre las cohortes de 1841-50 y 1911-20 fue sólo de 1,5 $\mathrm{cm}$. Hasta entonces, Castilla y León mostró unas estaturas por debajo de la media española y a gran distancia de otras regiones españolas. Para las generaciones nacidas a partir de 1930 (reemplazos de 1950) se produce una mayor convergencia con la estatura media de los españoles. El hecho podría estar relacionado con el modelo de especialización productiva y la emigración desencadenante hacia América y País Vasco, al haber perdido a los jóvenes varones más productivos por sus cualidades físicas. Durante el franquismo el bienestar biológico mejora en términos relativos, pero se advierte un incremento de los niveles de pobreza y de los cortos de talla en los reemplazos a comienzos de la década de 1950.

El crecimiento económico castellano afectó de manera muy desigual a los pobladores de la Meseta, en función de su estatus social y de su residencia. Durante buena parte del siglo XIX, el deterioro de los niveles de vida fue mucho más ostensible en las ciudades y entre la población jornalera. En el siglo XX, la población rural ocupada en las tareas del campo (con excepción de las que habitaban en las zonas de montaña económicamente más dinámicas, Moreno 2005b) sufrió mayores penalidades, a las que ni siquiera los propietarios de pequeñas explotaciones fueron ajenos.

En general, los resultados muestran que las estaturas de los mozos llamados a reclutamiento son de enorme utilidad para el estudio del bienestar humano y de los niveles de vida en la España contemporánea, al manifestarnos la relación que se establece entre talla, enfermedad, infección, trabajo infantil, renta y condiciones ambientales. La estatura es un reflejo de las condiciones de vida biológicas y un verdadero proxy de la desigualdad socioeconómica.

Recibido: 30-06-2008

Aceptado: 27-11-2008 